Pacific

Journal of

Mathematics

RELATIVE FORMULF FOR THE SPECTRAL INVARIANTS OF THE $b$-CALCULUS AND GENERALIZED APS BOUNDARY PROBLEMS OF DIRAC OPERATORS

PAUl Loya AND JinSUNG PARK

Volume $223 \quad$ No. 2

February 2006 


\title{
RELATIVE FORMUL $F$ FOR THE SPECTRAL INVARIANTS OF THE $b$-CALCULUS AND GENERALIZED APS BOUNDARY PROBLEMS OF DIRAC OPERATORS
}

\author{
PAUL LOYA AND JiNSUNG PARK
}

\begin{abstract}
We relate zeta determinants of Dirac operators with generalized APS boundary conditions for compact manifolds with boundary and parallel $b$-zeta determinants of perturbed Dirac operators on the corresponding complete manifolds with cylindrical end. We also derive, without invertibility conditions, corresponding relative formulæ for the $(b-)$ zeta determinants.
\end{abstract}

\section{Introduction}

Boundary problems of generalized APS type have special geometric and topological importance, as can be seen in the Atiyah-Patodi-Singer index theorem (see Section 1A). In [Atiyah et al. 1975], those authors found a connection between their theorem and the index of the Dirac operator on a corresponding manifold with cylindrical end. Melrose [1993] worked out this connection and developed the $b$-calculus to give his "direct proof" of the APS index theorem. Recently, both the index and the eta invariant of Dirac operators with generalized APS conditions for manifolds with boundary were connected with parallel invariants of associated perturbed Dirac operators on the corresponding manifolds with cylindrical end; see [Melrose and Piazza 1997; Loya and Melrose 2003; Loya 2005]. Here we derive a similar connection for the $\zeta$-determinant. To motivate this connection, we begin by reviewing the connection between the $b$-calculus and boundary value problems for the index and the eta invariant.

1A. The b-calculus and the index theorem. We first state our assumptions. Let D : $C^{\infty}(X, E) \longrightarrow C^{\infty}(X, F)$ be a compatible Dirac type operator associated to a $\mathbb{Z}_{2}$-graded Hermitian Clifford module $E \oplus F$ over a compact Riemannian manifold $X$ with boundary $Y$. We assume that all the geometric structures are of product type on a collar $[-1,0]_{u} \times Y$ of the boundary $\{u=0\}=\partial X=Y$. Therefore, on this collar we assume that $\left.E \cong E\right|_{u=0},\left.F \cong F\right|_{u=0}$, and

$$
\mathscr{D}=G\left(\partial_{u}+D_{Y}\right) \text {, }
$$

MSC2000: primary 58J28; secondary 58J35, 58J20, $58 \mathrm{~J} 32$.

Keywords: Dirac operators, $b$-calculus, $\zeta$-determinants. 

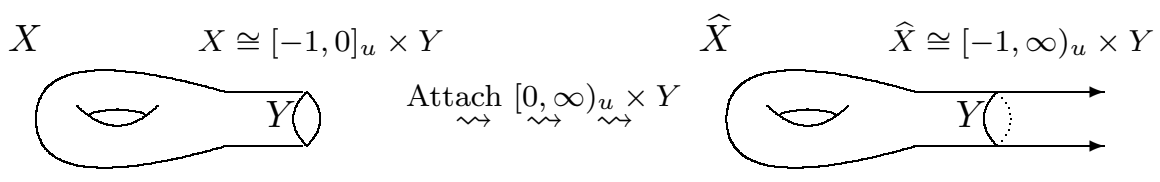

Figure 1. Creating a manifold with cylindrical end.

where $G:\left.\left.E\right|_{u=0} \longrightarrow F\right|_{u=0}$ is a unitary isomorphism and $D_{Y}$ is a Dirac operator on $Y$. Let $\Pi_{+}, \Pi_{-}$, and $\Pi_{0}$ denote the orthogonal projections of $L^{2}\left(Y, E_{0}\right)$, where $E_{0}:=\left.E\right|_{u=0}$, onto the positive, negative, and zero eigenspaces, respectively, of $D_{Y}$.

Let us assume for the moment that $X$ is even-dimensional. Let $T$ be a unitary involution (that is, $T^{2}=\mathrm{Id}$ ) on $V:=\operatorname{ker} D_{Y}$. Then $T$ has \pm 1 eigenvalues. We define $\mathscr{D}_{T}$ as the Dirac operator $\mathscr{D}$ with domain

$$
\operatorname{dom}\left(\mathscr{D}_{T}\right):=\left\{\phi \in H^{1}(X, E) \mid \Pi_{-}^{T}\left(\left.\phi\right|_{u=0}\right)=0\right\},
$$

where $\Pi_{-}^{T}:=\Pi_{-}+\Pi_{-T}$ with $\Pi_{L}:=\frac{1}{2}(\mathrm{Id}+L) \Pi_{0}$ for any involution $L$ on $V$. Such a boundary condition is called a generalized APS boundary condition.

Let $\widehat{X}$ be the manifold formed by gluing the infinite cylinder $[0, \infty)_{u} \times Y$ to the end of the collar $[-1,0]_{u} \times Y$ of $X$ (see Figure 1):

$$
\widehat{X}:=X \sqcup_{\partial X}\left([0, \infty)_{u} \times Y\right) .
$$

All the geometric structures on $X$ extend naturally to the manifold $\widehat{X}$. We use the same notations for these extended objects on $\widehat{X}$ as for the original objects on $X$, except we denote the extended Dirac operator by $\widehat{\mathscr{D}}$.

Given a self-adjoint involution $T$ on $V$, following [Melrose and Piazza 1997], we show in Section 2 how to construct a corresponding $b$-smoothing operator $\widehat{T} \in$ $\Psi_{b}^{-\infty}(\widehat{X}, E, F)$ such that the $L^{2}$ based operator

$$
\widehat{\mathscr{D}}+\widehat{T}: H^{1}(\widehat{X}, E) \longrightarrow L^{2}(\widehat{X}, F)
$$

is "linked" to the operator $\mathscr{D}_{T}$ on the compact manifold $X$. More precisely, in [Melrose and Piazza 1997; Loya 2005], the index theoretic properties of $X$ and $\widehat{X}$ were linked as follows. The operators $\widehat{\mathscr{D}}+\widehat{T}$ and $\mathscr{D}_{T}$ have the same index theoretic properties:

(a) $\operatorname{ker}(\widehat{D}+\widehat{T}) \cong \operatorname{ker} \mathscr{D}_{T}$ and $\operatorname{ker}(\widehat{\mathscr{D}}+\widehat{T})^{*} \cong \operatorname{ker}\left(\mathscr{D}_{T}\right)^{*}$.

(b) $\widehat{D}+\widehat{T}: H^{1}(\widehat{X}, E) \longrightarrow L^{2}(\widehat{X}, F)$ and $\mathscr{D}_{T}: \operatorname{dom}\left(\mathscr{D}_{T}\right) \longrightarrow L^{2}(X, F)$ are Fredholm with equal indices (by (a)). 
(c) The following index formula holds:

$$
\operatorname{ind}(\widehat{D}+\widehat{T})=\operatorname{ind} \mathscr{D}_{T}=\int_{X} \mathrm{AS}+\frac{1}{2}\left(\eta\left(D_{Y}\right)+\operatorname{sign} T\right),
$$

where AS is the Atiyah-Singer index density and where $\eta\left(D_{Y}\right)$ is the eta invariant of $D_{Y}$ (see Section 1B). Note that $Y$ is a "right boundary"; this accounts for the + instead of - in front of the eta term.

The connections (b) and (c) were first observed in [Melrose and Piazza 1997]. This theorem holds even when $\operatorname{dim} X$ is odd, but in this case $\int_{X}$ AS vanishes. As a trivial corollary, we get the following relative formula: for any two such maps $T$ and $S$ on $V$, we have

$$
\operatorname{ind}(\widehat{D}+\widehat{T})-\operatorname{ind}(\widehat{\mathscr{D}}+\widehat{S})=\operatorname{ind} \mathscr{D}_{T}-\operatorname{ind} \mathscr{D}_{S}=\frac{1}{2}(\operatorname{sign} T-\operatorname{sign} S) .
$$

1B. The b-calculus and the eta invariant. We now review the connection between the eta invariants of $\widehat{\mathscr{D}}+\widehat{T}$ and $\mathscr{D}_{T}$ established in [Loya 2005; Loya and Melrose 2003]. Henceforth we assume that $E=F$ and $X$ can be of arbitrary dimension. Then $G$ is a unitary isomorphism on $E_{0}$ only, since $E=F$. Moreover, Clifford algebra and self-adjointness considerations impose the relations

$$
G^{2}=-\mathrm{Id}, \quad G^{*}=-G, \quad G D_{Y}=-D_{Y} G .
$$

The last of these equalities implies that $G$ acts on $V$. We denote by $\mathscr{L}(V)$ the set of unitary involutions $T$ on $V$ such that $G T=-T G$; for such a $T$, we denote the +1 eigenspace of $T$ by $\Lambda_{T}$. There is a distinguished subspace $\Lambda_{\sigma}$ of $V$ defined by

$$
\Lambda_{\sigma}:=\left\{\Pi_{0}\left(\left.\phi\right|_{u=0}\right) \mid \phi \in H^{1}(X, E), \mathscr{D} \phi=0, \Pi_{-}\left(\left.\phi\right|_{u=0}\right)=0\right\} .
$$

If $\Pi_{\sigma}$ is the orthogonal projection onto $\Lambda_{\sigma}$, then $\sigma:=2 \Pi_{\sigma}-\mathrm{Id}$, acting on $V$, is in $\mathscr{L}(V)$ with associated +1 eigenspace $\Lambda_{\sigma}$. The unitary map $\sigma$ is called the scattering matrix and $\Lambda_{\sigma}$ is called the scattering Lagrangian.

We now recall the definition of the eta invariant of $\mathscr{D}_{T}$ for $T \in \mathscr{L}(V)$; compare [Douglas and Wojciechowski 1991; Grubb and Seeley 1996]. Since $T \in \mathscr{L}(V)$, it turns out that the operator $\mathscr{D}_{T}$ is self-adjoint and has real discrete spectrum. If $\left\{\lambda_{j}\right\}$ are the eigenvalues of $\mathscr{D}_{T}$, then the eta function of $\mathscr{D}_{T}$,

$$
\eta_{\mathscr{D}_{T}}(s):=\sum_{\lambda_{j} \neq 0} \frac{\operatorname{sign} \lambda_{j}}{\left|\lambda_{j}\right|^{s}},
$$

extends from $\operatorname{Re} s \gg 0$ to be a meromorphic function of $s \in \mathbb{C}$ that is regular at $s=0$. The eta invariant is by definition $\eta\left(\mathscr{D}_{T}\right):=\eta_{\mathscr{D}_{T}}(0)$.

The operator $\widehat{\mathscr{D}}+\widehat{T}$ over the noncompact manifold $\widehat{X}$ has a corresponding invariant, called the $b$-eta invariant, which we denote by ${ }^{b} \eta(\widehat{\mathscr{D}}+\widehat{T})$. In [Loya 2005; Loya and Melrose 2003], the eta invariant theoretic properties of $X$ and $\widehat{X}$ were 
linked as follows (for odd-dimensional manifolds, but the even-dimensional case is similar). For $T \in \mathscr{L}(V)$, the perturbed operator $\widehat{\mathscr{D}}+\widehat{T}$ in $(1-2)$ and the operator $\mathscr{D}_{T}$ with domain (1-1) have the same eta invariant theoretic properties:

(a) ${ }^{b} \eta(\widehat{D}+\widehat{T})=\eta\left(\mathscr{D}_{T}\right)$.

(b) The following formula holds:

$$
{ }^{b} \eta(\widehat{D}+\widehat{T})=\eta\left(\mathscr{D}_{T}\right)=\eta\left(\mathscr{D}_{-\sigma}\right)+m\left(\Lambda_{T}, \Lambda_{\sigma}\right),
$$

where $\sigma$ is the scattering matrix.

Here, the " $m$-function" was introduced in [Lesch and Wojciechowski 1996] (compare [Bunke 1995]): For $T, S \in \mathscr{L}(V)$,

$$
m\left(\Lambda_{T}, \Lambda_{S}\right):=-\frac{1}{i \pi} \sum_{\substack{e^{i \theta} \in \operatorname{spec}(-[(i+G) / 2 i] T S) \\ \theta \in(-\pi, \pi)}} i \theta .
$$

As a trivial corollary, we get the following relative formula:

$$
{ }^{b} \eta(\widehat{D}+\widehat{T})-{ }^{b} \eta(\widehat{D}-\widehat{\sigma})=\eta\left(\mathscr{D}_{T}\right)-\eta\left(\mathscr{D}_{-\sigma}\right)=m\left(\Lambda_{T}, \Lambda_{\sigma}\right) .
$$

This formula is related to the gluing problem for the ( $b$-)eta invariant, which has been investigated by many authors; see for instance [Dai and Freed 1994; Bunke 1995; Mazzeo and Melrose 1995; Hassell et al. 1995; Wojciechowski 1995; Müller 1996; Brüning and Lesch 1999; Kirk and Lesch 2004; Loya and Park 2006; [2005]], and see also the survey article [Mazzeo and Piazza 1998] for more on this topic.

1C. The b-calculus and the $\zeta$-determinant. To paraphrase the previous two sections: $\widehat{\mathscr{D}}+\widehat{T}$ and $\mathscr{D}_{T}$ have identical index and eta invariant theoretic properties; moreover, we have exact (no integer ambiguities) relative invariant formulæ (1-3) and $(1-4)$. The purpose of this paper is to investigate the $\zeta$-determinant connection of $X$ and $\widehat{X}$, which we now explain. Recall that if $\left\{\lambda_{j}\right\}$ are the eigenvalues of $\mathscr{D}_{T}$, then the zeta function of $\mathscr{D}_{T}^{2}:=\left(\mathscr{D}_{T}\right)^{2}$ is

$$
\zeta_{\mathscr{D}_{T}^{2}}(s):=\sum_{\lambda_{j} \neq 0} \lambda_{j}^{-2 s},
$$

which is defined a priori for $\operatorname{Re} s \gg 0$ and has an analytic continuation to the whole complex plane with 0 as a regular point [Grubb and Seeley 1996]. Then the $\zeta$-determinant of $\mathscr{D}_{T}^{2}$ is by definition

$$
\operatorname{det}_{\zeta} \mathscr{D}_{T}^{2}:=\exp \left(-\left.\frac{d}{d s}\right|_{s=0} \zeta_{\mathscr{D}_{T}^{2}}(s)\right) .
$$

Since $(\widehat{D}+\widehat{T})^{2}$ has discrete and continuous spectrum, it does not have a $\zeta$-function as in (1-5). However, there is a natural generalization called the $b$-zeta function 
$b_{\zeta_{(\widehat{D}+\widehat{T})^{2}}}(s)$ (see Section 2) and then the $b$-zeta determinant $\operatorname{det}_{b_{\zeta}}(\widehat{\mathscr{D}}+\widehat{T})^{2}$ can be defined in terms of $b_{\zeta(\widehat{D}+\widehat{T})^{2}}(s)$ by the formula (1-6).

Now the question arises: Given $T \in \mathscr{L}(V)$, what is the "defect" of

$$
\frac{\operatorname{det}_{\zeta}(\widehat{D}+\widehat{T})^{2}}{\operatorname{det}_{\zeta} \mathscr{D}_{T}^{2}}=?
$$

One may conjecture that there is no defect (that is, the ratio is unity) in view of the fact that there are no "defects" for the index and the eta invariant. The first main result of this paper shows that this is not the case. To detail this theorem, recall that $\left.\operatorname{ker}(\widehat{\mathscr{D}}+\widehat{T})\right|_{X} \equiv \operatorname{ker} \mathscr{D}_{T}$. On the finite-dimensional vector space $\gamma_{0} \operatorname{ker}(\widehat{\mathscr{D}}+\widehat{T})=$ $\gamma_{0} \operatorname{ker} \mathscr{D}_{T}$, where $\gamma_{0}$ is the restriction map from $X$ to $\{u=0\}$, we define

$$
\widehat{\mathscr{L}}_{T}:=\sum \gamma_{0} \widehat{\phi}_{k} \otimes \gamma_{0} \widehat{\phi}_{k}, \quad \mathscr{L}_{T}:=\sum \gamma_{0} \phi_{k} \otimes \gamma_{0} \phi_{k},
$$

where $\left\{\widehat{\phi}_{k}\right\}$ and $\left\{\phi_{k}\right\}$ are orthonormal bases for the kernels of $\widehat{\mathscr{D}}+\widehat{T}$ and $\mathscr{D}_{T}$, respectively. We can now state our first result.

Theorem 1.1. For any $T \in \mathscr{L}(V)$, the perturbed Dirac operator $\widehat{\mathscr{D}}+\widehat{T}$ and the operator $\mathscr{D}_{T}$ have the following relation:

(1-7) $\frac{\operatorname{det}_{\zeta}(\widehat{\mathscr{D}}+\widehat{T})^{2}}{\operatorname{det}_{\zeta} \mathscr{D}_{T}^{2}}=2^{-\frac{1}{2} \zeta_{D_{Y}^{2}}(0)}\left(\frac{\operatorname{det} \widehat{\mathscr{L}}_{T}}{\operatorname{det} \mathscr{L}_{T}}\right)^{-2}\left(\frac{\left(\operatorname{det}_{\zeta} \Delta_{d}\right)\left(\operatorname{det}_{b_{\zeta}} \Delta_{v}\right)}{4}\right)^{h_{Y} / 2}$,

where $h_{Y}=\operatorname{dim} \operatorname{ker} D_{Y}, \zeta_{D_{Y}^{2}}(s)$ is the $\zeta$-function of $D_{Y}^{2}$, and where $\Delta_{d}$ and $\Delta_{v}$ are perturbed one-dimensional Dirichlet and Neumann Laplacians (defined independently of $T \in \mathscr{L}(V)$ ), respectively, acting on scalar functions over the half-line $[0, \infty)$; see $(3-2)$.

We remark that the value of the right-hand side of (1-7) varies with $T$ and is maximized when $T=\sigma$. To see this, by Proposition 4.1, we have

$$
\left(\frac{\operatorname{det} \widehat{\mathscr{L}}_{T}}{\operatorname{det} \mathscr{L}_{T}}\right)^{-2}=\left(\prod_{k=1}^{\operatorname{dim} \operatorname{ker}(\widehat{\mathscr{D}}+\widehat{T})} \int_{X}\left|\widehat{\phi}_{k}\right|^{2} d g\right)^{-2} .
$$

By Theorem 2.2, $\operatorname{dim} \operatorname{ker}(\widehat{\mathscr{D}}+\widehat{T})=\operatorname{dim}\left(\Lambda_{T} \cap \Lambda_{\sigma}\right)+\operatorname{dim} \operatorname{ker} \mathscr{D}_{-\sigma}$, so the value of (1-8) can be changed by varying $T$ so that the number of elements of the intersection $\Lambda_{T} \cap \Lambda_{\sigma}$ increases or decreases. In particular, (1-8) has a maximum when $T=\sigma$ since $\int_{X}\left|\widehat{\phi}_{k}\right|^{2} d g<1$ (because $\int_{\widehat{X}}\left|\widehat{\phi}_{k}\right|^{2} d g=1$ ). Therefore, the right-hand side of (1-7) can vary with $T$ and is maximized when $T=\sigma$, since $2^{-\zeta_{D_{Y}^{2}}}(0) / 2$ and $\operatorname{det}_{b_{\zeta}} \Delta_{d} \cdot \operatorname{det}_{b_{\zeta}} \Delta_{v}$ are independent of $T$.

We now extend the relative formulæ $(1-3)$ and $(1-4)$ to the $\zeta$-determinant. For a linear operator $L$ over a finite-dimensional vector space, $\operatorname{set} \operatorname{det}^{*} L:=\left.\operatorname{det} L\right|_{(\operatorname{ker} L)^{\perp}}$. Our second main result is the following relative formula. 


$$
\begin{gathered}
\operatorname{ind}(\widehat{\mathscr{D}}+\widehat{T})=\text { ind } \mathscr{D}_{T} \\
{ }_{\eta}(\widehat{\mathscr{D}}+\widehat{T})=\eta\left(\mathscr{D}_{T}\right) \\
\frac{\operatorname{det}_{\zeta}(\widehat{\mathscr{D}}+\widehat{T})^{2}}{\operatorname{det}_{\zeta} \mathscr{D}_{T}^{2}}=2^{-\frac{1}{2} \zeta_{D_{Y}^{2}}(0)}\left(\frac{\operatorname{det} \widehat{\mathscr{L}}_{T}}{\operatorname{det} \mathscr{L}_{T}}\right)^{-2}\left(\frac{\left(\operatorname{det}_{\zeta} \Delta_{d}\right)\left(\operatorname{det}_{\zeta} \Delta_{\nu}\right)}{4}\right)^{h_{Y} / 2}
\end{gathered}
$$

Table 1. $b$-calculus and BVP relative formulæ (no integer ambiguities).

Theorem 1.2. Given $T \in \mathscr{L}(V)$, the following formulas hold:

$$
\begin{aligned}
\frac{\operatorname{det}_{\zeta}(\widehat{\mathscr{D}}+\widehat{T})^{2}}{\operatorname{det}_{b_{\zeta}}(\widehat{\mathscr{D}}-\widehat{\sigma})^{2}} & =\left(\frac{\operatorname{det} \widehat{\mathscr{L}}_{T}}{\operatorname{det} \widehat{\mathscr{L}}_{-\sigma}}\right)^{-2} \operatorname{det}^{*}\left(\frac{2 \mathrm{Id}-\sigma^{+} T^{-}-T^{+} \sigma^{-}}{4}\right), \\
\frac{\operatorname{det}_{\zeta} \mathscr{D}_{T}^{2}}{\operatorname{det}_{\zeta} \mathscr{D}_{-\sigma}^{2}} & =\left(\frac{\operatorname{det} \mathscr{L}_{T}}{\operatorname{det} \mathscr{L}_{-\sigma}}\right)^{-2} \operatorname{det}^{*}\left(\frac{2 \mathrm{Id}-\sigma^{+} T^{-}-T^{+} \sigma^{-}}{4}\right) .
\end{aligned}
$$

In the case that $\operatorname{ker} \mathscr{D}_{T}=\operatorname{ker} \mathscr{D}_{-\sigma}=0$, the second formula in Theorem $1.2 \mathrm{can}$ be derived from [Scott 2002; Scott and Wojciechowski 2000]. We emphasize that the term $\left(\operatorname{det} \mathscr{L}_{-\sigma}\right)^{2} /\left(\operatorname{det} \mathscr{L}_{T}\right)^{2}$ in this formula is new, and this factor is nontrivial in general. For reference we summarize the relationships between the $b$-calculus and BVPs in Table 1.

Though the eta invariant and $\zeta$-determinant are nonlocal quantities, the $\zeta$-determinant is more nonlocal in the following variational sense (see [Atiyah et al. 1976, Propositions (2.9) and (2.10)]): the variation of the eta is "local" in that it only involves finitely many terms of the local symbol expansions of the original operator while the variation of the $\zeta$-determinant is "nonlocal" because the variation involves the inverse of the operator. With this in mind, we remark that the first two lines of Table 1 hold, basically, because the index and the variation of the eta invariant are "local" objects so these invariants are not able to distinguish between $X$ and $\widehat{X}$. Because the $\zeta$-determinant and its variation are "nonlocal", the last line of Table 1 shows that the $\zeta$-determinant is able to distinguish between $X$ and $\widehat{X}$.

This paper is organized as follows. In Section 2 we explicitly construct the $b$ smoothing perturbation $\widehat{T}$ corresponding to the matrix $T \in \mathscr{L}(V)$. In Section 3 we derive gluing formulæ for the $\zeta$-determinants on $X$ and $\widehat{X}$ from the gluing theorems proved in [Loya and Park 2006; 2005]. Lastly, in Section 4 we use these gluing theorems to prove Theorems 1.1 and 1.2. 


\section{Perturbed Dirac operators and the $b$-zeta function}

Let us henceforth fix $T \in \mathscr{L}(V)$. In this section, we construct the perturbation $\widehat{T}$ in Theorem 1.1 and we review the $b$-trace and the $b$-zeta determinant.

2A. Perturbations of Dirac operators. We first define an auxiliary $b$-smoothing operator acting on scalar functions on the half-line $[0, \infty)$. Let $\chi \in C^{\infty}(\mathbb{R})$ be a cut-off function, where $\chi(u)=1$ for $u \geq 2$ and $\chi(u)=0$ for $u \leq 1$. Let $\varrho \geq 0$ be a smooth compactly supported even function on $\mathbb{R}$ with $\varrho(0)>0$. Then $\widehat{\varrho}(\tau)$ is an even entire function - throughout the rest of this paper, the functions $\chi$ and $\varrho$ shall remain fixed. Define an operator $Q$ acting on Schwartz functions over $[0, \infty)$ by setting

$$
Q \psi:=\frac{1}{2 \pi} \chi(u) \int_{\mathbb{R}} e^{i u \tau} \widehat{\varrho}(\tau) \widehat{\chi \psi}(\tau) d \tau,
$$

where $\widehat{\chi \psi}$ is the Fourier transform of $\chi \psi$ :

$$
\widehat{\chi \psi}(\tau)=\int_{\mathbb{R}} e^{-i u \tau} \chi(u) \psi(u) d u .
$$

Since $\varrho$ is compactly supported, $\widehat{\varrho}(\tau)$ vanishes to infinite order as $|\tau| \rightarrow \infty$ for $|\operatorname{Im} \tau|$ within any fixed bound and therefore, $Q$ is by definition a $b$-pseudodifferential operator of order $-\infty$ (a " $b$-smoothing operator"). Moreover, since $\varrho$ is even, $\widehat{\varrho}(\tau)$ is also even, so $K_{Q}\left(u, u^{\prime}\right)=\overline{K_{Q}\left(u^{\prime}, u\right)}$, which implies that $Q$ is formally self-adjoint. Here is another of the main properties of $Q$ :

Lemma 2.1 [Loya 2005; Loya and Melrose 2003]. If W is a subspace of the kernel $V$ of $D_{Y}$ and $w \in W$ is arbitrary, the boundary value problem

$$
v \in H^{1}([0, \infty), V), \quad\left(\partial_{u}+Q^{2} T\right) v=0,\left.\quad v\right|_{u=0}=w,
$$

has a nontrivial solution if and only if $w \in \Lambda_{T} \cap W$, in which case the solution is unique and also takes values in $\Lambda_{T} \cap W$.

As in [Melrose and Piazza 1997], we define $\widehat{T}: L^{2}(\widehat{X}, E) \longrightarrow H^{\infty}(\widehat{X}, E)$, which is completely supported on the cylindrical end $[0, \infty)_{u} \times Y$, by

$$
\widehat{T}:=G Q^{2} T,
$$

where $Q$ is in (2-1). Note that $T$ is a finite rank smoothing operator acting over the cross section $Y$ while $Q$ acts over the half-line $[0, \infty)_{u}$. Then $\widehat{T} \in \Psi_{b}^{-\infty}(\widehat{X}, E)$, the space of $b$-pseudodifferential operator of order $-\infty$, by definition of this space [Loya 2005; Melrose 1993]. The following theorem, a consequence of Lemma 2.1, gives one of the main properties of the perturbation $\widehat{T}$. 
Theorem 2.2 [Loya 2005; Loya and Melrose 2003]. The operator

$$
\widehat{D}+\widehat{T}: H^{1}(\widehat{X}, E) \longrightarrow L^{2}(\widehat{X}, E)
$$

is Fredholm, and $\operatorname{ker}(\widehat{\mathscr{D}}+\widehat{T})$ is canonically isomorphic to $\operatorname{ker} \mathscr{D}_{T}$ by $\left.\operatorname{ker}(\widehat{D}+\widehat{T})\right|_{X} \equiv$ $\operatorname{ker} \mathscr{D}_{T}$. Moreover, these are canonically isomorphic to $\left(\Lambda_{T} \cap \Lambda_{\sigma}\right) \oplus \operatorname{ker} \mathscr{D}_{-\sigma}$.

We remark that the operator $\widehat{\mathscr{D}}$ is Fredholm if and only if $V=0$ [Melrose 1993], so $\widehat{D}$ alone is almost never Fredholm. As this theorem shows, the main impetus for introducing perturbations is to achieve the Fredholm property.

2B. The b-zeta function. Recall that the heat operator $e^{-t(\widehat{D}+\widehat{T})^{2}}$ is $b$-trace class [Melrose 1993, Chapter 4] with a long time expansion [Loya 2005; Loya and Melrose 2003; Melrose and Piazza 1997]:

$$
{ }^{b} \operatorname{Tr} e^{-t(\widehat{D}+\widehat{T})^{2}} \sim b_{0}+b_{1} e^{-\varepsilon t} \quad \text { as } t \rightarrow \infty,
$$

where $b_{0}=\operatorname{dim} \operatorname{ker}(\widehat{\mathscr{D}}+\widehat{T})$ and where $\varepsilon>0$. The reason for the exponential decay is that $\widehat{\mathscr{D}}+\widehat{T}$ is Fredholm so has discrete spectrum near 0. Also, there is the usual short time asymptotic expansion [Loya 2005; Loya and Melrose 2003; Melrose and Piazza 1997]:

$$
{ }^{b} \operatorname{Tr} e^{-t(\widehat{D}+\widehat{T})^{2}} \sim \sum_{k=0}^{\infty} a_{k} t^{k-\frac{n}{2}}+\sum_{k=1}^{\infty} a_{k}^{\prime} t^{k} \quad \text { as } t \rightarrow 0,
$$

where $n=\operatorname{dim} \widehat{X}$. Using this and (2-2), a straightforward computation shows that

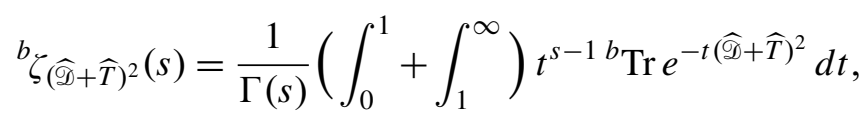

where the first integral is defined a priori for $\operatorname{Re} s \gg 0$ and the second one a priori for $\operatorname{Re} s \ll 0$, extend to be meromorphic functions on $\mathbb{C}$ that are regular at $s=0$. In particular, the $b$-zeta determinant

$$
\operatorname{det}_{b_{\zeta}}(\widehat{D}+\widehat{T})^{2}:=\exp \left(-\left.\frac{d}{d s} b_{\zeta(\widehat{D}+\widehat{T})^{2}}(s)\right|_{s=0}\right)
$$

is well-defined.

\section{Gluing formulæ for the $\zeta$-determinant}

In this section, applying the gluing formulae of the $\zeta$-determinant in [Loya and Park 2006; 2005], we prove two propositions which will be used in the proof of Theorems 1.1 and 1.2. 


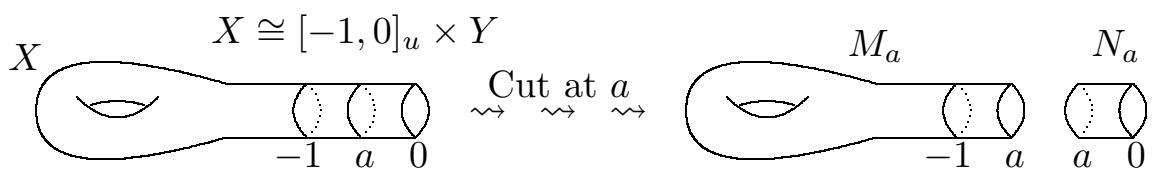

Figure 2. Cutting $X$ into two pieces.

3A. Gluing formula for $\boldsymbol{X}$. Let $-1<a<0$. We shall apply the gluing formula in [Loya and Park 2006, Theorem 1.1] to the operator $\mathscr{D}_{T}$ over the manifold $X$, which we decompose at $u=a$ :

$$
X=M_{a} \cup N_{a}, \quad M_{a}, N_{a}=\text { the left, right half of }\{u=a\} \text { in } X ;
$$

see Figure 2. Let $\mathscr{C}_{M_{a}}$ and $\mathscr{C}_{N_{a}}$ denote the Calderón projectors of $\left.\mathscr{D}_{T}\right|_{M_{a}}$ and $\left.\mathscr{D}_{T}\right|_{N_{a}}$, respectively, at the hypersurface $\{u=a\}$. Since $\mathscr{D}_{T}$ has the boundary condition $\Pi_{-}^{T}=\Pi_{-}+\frac{1}{2}(\operatorname{Id}-T) \Pi_{0}$ at $\{u=0\}$, one can check that $\mathscr{C}_{N_{a}}=\Pi_{+}^{-T}=$ $\Pi_{+}+\frac{1}{2}(\mathrm{Id}+T) \Pi_{0}$, which is independent of $a$. Thus, $\mathscr{D}_{\mathscr{C}_{N_{a}}}$ is just the operator $G\left(\partial_{u}+D_{Y}\right)$ over $[a, 0] \times Y$ with boundary conditions $\Pi_{+}^{-T}$ at $\{u=a\}$ and $\Pi_{-}^{T}$ at $\{u=0\}$. By [Loya and Park 2004, Theorem 1.1], we have

$$
\operatorname{det}_{\zeta} \mathscr{D}_{\mathscr{C}_{N_{a}}}^{2}=e^{-C a} 2^{\zeta_{D_{Y}^{2}}(0)+h_{Y}},
$$

where $C=-(2 \sqrt{\pi})^{-1}\left(\Gamma(s)^{-1} \Gamma\left(s-\frac{1}{2}\right) \zeta_{D_{Y}^{2}}\left(s-\frac{1}{2}\right)\right)^{\prime}(0)$. Now we recall that the Calderón projectors $\mathscr{C}_{M_{a}}, \Pi_{+}^{-T}$ have the forms

$$
\mathscr{C}_{M_{a}}=\frac{1}{2}\left(\begin{array}{cc}
\mathrm{Id} & \kappa_{a}^{-1} \\
\kappa_{a} & \mathrm{Id}
\end{array}\right), \quad \Pi_{+}^{-T}=\frac{1}{2}\left(\begin{array}{cc}
\mathrm{Id} & \kappa_{T}^{-1} \\
\kappa_{T} & \mathrm{Id}
\end{array}\right)
$$

with respect to $L^{2}\left(Y, E_{0}\right)=L^{2}\left(Y, E^{+}\right) \oplus L^{2}\left(Y, E^{-}\right)$where $E^{ \pm}$are the subbundles of $E_{0}$ consisting of the $( \pm i)$-eigensections of $G$. Let $U_{T, a}:=-\kappa_{a} \kappa_{T}^{-1}$, which is a unitary operator on $L^{2}\left(Y, E^{-}\right)$, and let $\widehat{U}_{T, a}$ denote the restriction of $U_{T, a}$ to the orthogonal complement of its (-1)-eigenspace. Finally let $\mathscr{L}_{T, a}:=\sum \gamma_{a} \phi_{k} \otimes \gamma_{a} \phi_{k}$ with $\gamma_{a}$ the restriction map from $X$ to $\{u=a\}$ and $\left\{\phi_{k}\right\}$ an orthonormal basis for $\operatorname{ker} \mathscr{D}_{T}$. Then by [Loya and Park 2006, Theorem 1.1] and (3-1), we obtain:

\section{Proposition 3.1.}

$$
\frac{\operatorname{det}_{\zeta} \mathscr{D}_{T}^{2}}{\operatorname{det}_{\zeta} \mathscr{D}_{\mathscr{C}_{M_{a}}}^{2}}=e^{-C a}\left(\operatorname{det} \mathscr{L}_{T, a}\right)^{-2} \operatorname{det}_{F}\left(\frac{2 \operatorname{Id}+\widehat{U}_{T, a}+\widehat{U}_{T, a}^{-1}}{4}\right) .
$$

3B. Gluing formula for $\widehat{\boldsymbol{X}}$. Again assume that $-1<a<0$. We now apply the preceding argument to $\widehat{\mathscr{D}}+\widehat{T}$ over $\widehat{X}$ separated at $u=a$ :

$$
\widehat{X}=M_{a} \cup \widehat{N}_{a}, \quad M_{a}, \widehat{N}_{a}=\text { the left, right half of }\{u=a\} \text { in } \widehat{X} .
$$




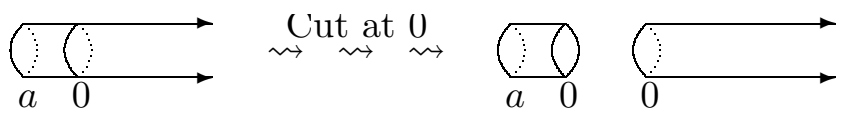

Figure 3. Cutting the cylinder.

Let $\mathscr{C}_{M_{a}}$ (just as before) and $\mathscr{C}_{\widehat{N}_{a}}$ denote the Calderón projectors of $\left.(\widehat{\mathscr{D}}+\widehat{T})\right|_{M_{a}}=$ $\left.\mathscr{D}\right|_{M_{a}}$ (since $\widehat{T}$ vanishes on $\left.X\right)$ and $\left.(\widehat{\mathscr{D}}+\widehat{T})\right|_{\widehat{N}_{a}}$ at $\{u=a\}$, respectively. Splitting $H^{1}([a, \infty) \times Y, E)$ into its projections onto $V=\operatorname{ker} D_{Y}$ and $V^{\perp}$, it follows that

$$
\left.(\widehat{D}+\widehat{T})\right|_{\widehat{N}_{a}}= \begin{cases}G\left(\partial_{u}+Q^{2} T\right) & \text { over } \Pi_{0} H^{1}([a, \infty) \times Y, E), \\ G\left(\partial_{u}+D_{Y}\right) & \text { over } \Pi_{0}^{\perp} H^{1}([a, \infty) \times Y, E) .\end{cases}
$$

Lemma 3.2. We have $\mathscr{C}_{\widehat{N}_{a}}=\Pi_{+}^{-T}$, which equals $\mathscr{C}_{N_{a}}$.

Proof. Lemma 2.1 immediately implies that $\mathscr{C}_{\widehat{N}_{a}}=\Pi_{+}^{-T}$ when $a=0$. To see that this holds even for the nonzero $a$, we recall that the statement of Lemma 2.1 is independent of the choice of $\chi, \varrho$, which define the operator $Q$ in (2-1). Therefore, we can show that the same statement is true for nonzero $a$ by shifting $\chi, \varrho$. Hence, the Calderón projector at $\{a\} \times Y$ is also given by $\Pi_{+}^{-T}$ even for nonzero $a$.

In the next proposition we compute $\operatorname{det}_{b_{\zeta}}(\widehat{D}+\widehat{T})_{\mathscr{C}_{\widehat{N}_{a}}}^{2}$.

Proposition 3.3. Over $H^{1}([0, \infty))$, define

$$
\begin{aligned}
& \Delta_{d}:=-\left(\partial_{u}-Q^{2}\right)\left(\partial_{u}+Q^{2}\right) \text { with Dirichlet condition at } u=0 \\
& \Delta_{v}:=-\left(\partial_{u}+Q^{2}\right)\left(\partial_{u}-Q^{2}\right) \text { with Neumann condition at } u=0 .
\end{aligned}
$$

These are one-dimensional "perturbed Laplace-type operators". Then

$$
\operatorname{det}_{\zeta}(\widehat{\mathscr{D}}+\widehat{T})_{\mathscr{C}_{\widehat{N}_{a}}}^{2}=\left(\left(\operatorname{det}_{b_{\zeta}} \Delta_{d}\right)\left(\operatorname{det}_{b_{\zeta}} \Delta_{\nu}\right)\right)^{h_{Y} / 2} \cdot e^{-C a} \cdot 2^{\frac{1}{2} \zeta_{D_{Y}^{2}}(0)} .
$$

Proof. We apply [Loya and Park 2005, Theorem 1.1] to $\operatorname{det}_{b_{\zeta}}(\widehat{\mathscr{D}}+\widehat{T})_{\mathscr{C}_{\widehat{N}_{a}}}^{2}$ with the decomposition

$$
[a, \infty) \times Y=([a, 0] \times Y) \cup([0, \infty) \times Y) .
$$

(see Figure 3). By Lemmas 2.1 and 3.2, it follows that the Calderón projectors at the left and right side of the dividing hypersurface $\{0\} \times Y$ are $\Pi_{-}^{T}$ and $\Pi_{+}^{-T}$, respectively. In particular, the induced operator on $[a, 0] \times Y$ is just the operator $G\left(\partial_{u}+D_{Y}\right)$ over $[a, 0] \times Y$ with boundary conditions $\Pi_{+}^{-T}$ at $\{u=a\}$ and $\Pi_{-}^{T}$ at $\{u=0\}$, which has $\zeta$-determinant equal to

$$
e^{-C a} 2^{\zeta_{D_{Y}^{2}}(0)+h_{Y}}
$$


see (3-1). Combining this value and [Loya and Park 2005, Theorem 1.1], one can derive

$$
\operatorname{det}_{b_{\zeta}}(\widehat{\mathscr{D}}+\widehat{T})_{\mathscr{C}_{\widehat{N}_{a}}}^{2}=e^{-C a} \cdot \operatorname{det}_{b_{\zeta}}(\widehat{\mathscr{D}}+\widehat{T})_{\Pi_{+}^{-T}}^{2} .
$$

It remains to compute $\operatorname{det}_{b_{\zeta}}(\widehat{D}+\widehat{T})_{\Pi_{+}^{-T}}^{2}$ on $[0, \infty) \times Y$. To do so, we observe that

$$
\operatorname{det}_{b_{\zeta}}(\widehat{D}+\widehat{T})_{\Pi_{+}^{-T}}^{2}=\operatorname{det}_{\zeta} A^{2} \cdot \operatorname{det}_{b_{\zeta}} B^{2},
$$

where

$$
\begin{array}{ll}
A:=G\left(\partial_{u}+Q^{2} T\right)_{\Pi_{T}} & \text { over } \Pi_{0} H^{1}([0, \infty) \times Y, E), \\
B:=G\left(\partial_{u}+D_{Y}\right)_{\Pi_{+}} & \text {over } \Pi_{0}^{\perp} H^{1}([0, \infty) \times Y, E) .
\end{array}
$$

By [Loya and Park 2005, Lemma 2.2], we have $\operatorname{det}_{\zeta} B^{2}=2^{\frac{1}{2} \zeta_{D_{Y}^{2}}(0)}$, so it now remains to compute $\operatorname{det}_{b_{\zeta}} A^{2}$. To this end, we recall that

$$
\operatorname{dom}(A)=\left\{\phi \in H^{1}([0, \infty), V) \mid \Pi_{T}\left(\left.\phi\right|_{u=0}\right)=0\right\},
$$

so

$$
\operatorname{dom}\left(A^{2}\right)=\left\{\phi \in H^{2}([0, \infty), V) \mid \Pi_{T}\left(\left.\phi\right|_{u=0}\right)=0, \Pi_{T}\left(\left.A \phi\right|_{u=0}\right)=0\right\} .
$$

Now the heat operator $e^{-t A^{2}}$ takes an initial condition $\psi$ to a function $\phi_{t}$ that satisfies

$$
\left(\partial_{t}+A^{2}\right) \phi_{t}=0 \quad ; \quad \phi_{0}=\psi \quad, \quad \Pi_{T}\left(\left.\phi_{t}\right|_{u=0}\right)=0 \quad \Pi_{T}\left(\left.A \phi_{t}\right|_{u=0}\right)=0 .
$$

Near $u=0, Q=0$, so at $u=0$ we have $\Pi_{T} A=\Pi_{T} G \partial_{u}=G \Pi_{-T} \partial_{u}$. Thus,

$$
\left(\partial_{t}+A^{2}\right) \phi_{t}=0 \quad ; \quad \phi_{0}=\psi \quad, \quad \Pi_{T}\left(\left.\phi_{t}\right|_{u=0}\right)=0 \quad, \quad \Pi_{-T}\left(\left.\partial_{u} \phi_{t}\right|_{u=0}\right)=0 .
$$

Since $\Pi_{T}$ is the orthogonal projection onto $\Lambda_{T}$ and $\Pi_{-T}$ is the one onto $\Lambda_{T}^{\perp}$, we have Dirichlet conditions on $\Lambda_{T}$ and Neumann conditions on $\Lambda_{T}^{\perp}$. Moreover,

$$
\begin{aligned}
A^{2}=G\left(\partial_{u}+Q^{2} T\right) G\left(\partial_{u}+Q^{2} T\right) & =-\left(\partial_{u}-Q^{2} T\right)\left(\partial_{u}+Q^{2} T\right) \\
& = \begin{cases}-\left(\partial_{u}-Q^{2}\right)\left(\partial_{u}+Q^{2}\right) & \text { over } \Lambda_{T} \\
-\left(\partial_{u}+Q^{2}\right)\left(\partial_{u}-Q^{2}\right) & \text { over } \Lambda_{T}^{\perp} .\end{cases}
\end{aligned}
$$

Thus, by definition of $\Delta_{d}$ and $\Delta_{v}$,

$$
e^{-t A^{2}}=e^{-t \Delta_{d}} \Pi_{T}+e^{-t \Delta_{v}} \Pi_{-T} .
$$

Since $\operatorname{Tr} \Pi_{T}=\operatorname{dim} \Lambda_{T}=h_{Y} / 2$ with the same for $\operatorname{Tr} \Pi_{-T}$, we obtain

$$
b_{\zeta_{A^{2}}}(s)=\frac{h_{Y}}{2}\left(b_{\zeta_{\Delta_{d}}}(s)+{ }^{b_{\zeta_{\nu}}}(s)\right) \text {. }
$$

Using the definition of the $b$-zeta determinant finishes the proof. 
Again applying [Loya and Park 2005, Theorem 1.1] together with Proposition 3.3, we have:

\section{Proposition 3.4.}

$$
\begin{array}{r}
\frac{\operatorname{det}_{\zeta}(\widehat{D}+\widehat{T})^{2}}{\operatorname{det}_{\zeta} \mathscr{D}_{\mathscr{C}_{M_{a}}}^{2}}=e^{-C a} 2^{-\frac{1}{2} \zeta_{D_{Y}^{2}}(0)} \cdot\left(\frac{\left(\operatorname{det}_{\zeta} \Delta_{d}\right)\left(\operatorname{det}_{\zeta} \Delta_{\nu}\right)}{4}\right)^{P_{Y} / 2} \\
\cdot\left(\operatorname{det} \widehat{\mathscr{L}}_{T, a}\right)^{-2} \cdot \operatorname{det}_{F}\left(\frac{2 \mathrm{Id}+\widehat{U}_{T, a}+\widehat{U}_{T, a}^{-1}}{4}\right),
\end{array}
$$

where $\widehat{\mathscr{L}}_{T, a}:=\sum \gamma_{a} \widehat{\phi}_{k} \otimes \gamma_{a} \widehat{\phi}_{k}$ with $\left\{\widehat{\phi}_{k}\right\}$ an orthonormal basis for $\operatorname{ker}(\widehat{D}+\widehat{T})$.

\section{Proof of main theorems}

In this final section we put together the results obtained in the previous section to prove Theorems 1.1 and 1.2.

First of all, Theorem 1.1 is easy to prove: dividing the formulas in Propositions 3.1 and 3.4 , we obtain

$$
\frac{\operatorname{det}_{b_{\zeta}}(\widehat{\mathscr{D}}+\widehat{T})^{2}}{\operatorname{det}_{\zeta} \mathscr{D}_{T}^{2}}=2^{-\frac{1}{2} \zeta_{D_{Y}^{2}}(0)}\left(\frac{\operatorname{det} \widehat{\mathscr{L}}_{T, a}}{\operatorname{det} \mathscr{L}_{T, a}}\right)^{-2}\left(\frac{\left(\operatorname{det}_{\zeta} \Delta_{d}\right)\left(\operatorname{det}_{\zeta} \Delta_{\nu}\right)}{4}\right)^{h_{Y} / 2} .
$$

It follows that the ratio $\operatorname{det} \widehat{\mathscr{L}}_{T, a} / \operatorname{det} \mathscr{L}_{T, a}$ does not depend on $a$. In particular, we can take $a \rightarrow 0$ in this equality, which completes the proof of Theorem 1.1.

Theorem 1.2 takes a little more work. By Theorem 1.1, we can derive the ratio of $\operatorname{det}_{b_{\zeta}}(\widehat{\mathscr{D}}+\widehat{T})^{2}$ with $\operatorname{det}_{\zeta}(\widehat{\mathscr{D}}-\widehat{\sigma})^{2}$ from the ratio of $\operatorname{det}_{\zeta} \mathscr{D}_{T}^{2}$ with $\operatorname{det}_{\zeta} \mathscr{D}_{-\sigma}^{2}$, so we shall focus on the latter ratio. Applying Proposition 3.1 to $T$ and $-\sigma$, then dividing the resulting formulas, we obtain

$\frac{\operatorname{det}_{\zeta} \mathscr{D}_{T}^{2}}{\operatorname{det}_{\zeta} \mathscr{D}_{-\sigma}^{2}}=\left(\frac{\operatorname{det} \mathscr{L}_{T, a}}{\operatorname{det} \mathscr{L}_{-\sigma, a}}\right)^{-2} \operatorname{det}_{F}\left(\left(\frac{2 \mathrm{Id}+\widehat{U}_{T, a}+\widehat{U}_{T, a}^{-1}}{4}\right)\left(\frac{2 \mathrm{Id}+\widehat{U}_{-\sigma, a}+\widehat{U}_{-\sigma, a}^{-1}}{4}\right)^{-1}\right)$.

We can find the right-hand determinant as follows. First, we can write

$$
L^{2}\left(Y, E^{-}\right)=V^{-} \oplus\left(V^{\perp}\right)^{-},
$$

where $\left(V^{\perp}\right)^{-}=\frac{\mathrm{Id}+i G}{2} V^{\perp}$; this allows us to consider the above determinant over $V^{-}$and $\left(V^{\perp}\right)^{-}$separately. Second, we notice that

$$
\mathscr{C}_{M_{a}}=\frac{\mathrm{Id}+\sigma}{2} \text { over } V \Longrightarrow \kappa_{M_{a}}=\sigma^{+} \text {over } V^{+}
$$

because the intersection of the Cauchy data space with $V$ does not change with respect to $a$ by the description of the dynamics of the Cauchy data space in [Nicolaescu 1995]. Hence, by the definition of $U_{T, a}$ and $U_{-\sigma, a}$, we have

$$
U_{T, a}=-\sigma^{+} T^{-}, \quad U_{-\sigma, a}=-\sigma^{+}\left(-\sigma^{-}\right)=\mathrm{Id} \quad \text { over } V^{-} .
$$


Third, since $\Pi_{+}^{-T}=\Pi_{+}^{\sigma}$ (which is equal to $\Pi_{+}$) over $V^{\perp}$, it follows that

$$
U_{T, a}=U_{-\sigma, a} \text { over }\left(V^{\perp}\right)^{-} .
$$

Hence,

$\operatorname{det}_{F}\left(\frac{2 \mathrm{Id}+\widehat{U}_{T, a}+\widehat{U}_{T, a}^{-1}}{4}\right)\left(\frac{2 \mathrm{Id}+\widehat{U}_{-\sigma, a}+\widehat{U}_{-\sigma, a}^{-1}}{4}\right)^{-1}=\operatorname{det}^{*}\left(\frac{2 \mathrm{Id}-\sigma^{+} T^{-}-T^{+} \sigma^{-}}{4}\right)$, since $\left(\sigma^{+} T^{-}\right)^{-1}=T^{+} \sigma^{-}$. Thus,

$$
\frac{\operatorname{det}_{\zeta} \mathscr{D}_{T}^{2}}{\operatorname{det}_{\zeta} \mathscr{D}_{-\sigma}^{2}}=\left(\frac{\operatorname{det} \mathscr{L}_{T, a}}{\operatorname{det} \mathscr{L}_{-\sigma, a}}\right)^{-2} \cdot \operatorname{det}^{*}\left(\frac{2 \mathrm{Id}-\sigma^{+} T^{-}-T^{+} \sigma^{-}}{4}\right) .
$$

It follows that the ratio $\operatorname{det} \mathscr{L}_{T, a} / \operatorname{det} \mathscr{L}_{-\sigma, a}$ does not depend on $a$. In particular, we can take $a \rightarrow 0$ in this equality, which completes our proof of Theorem 1.2.

Finally, we end our paper with a proof of the following "explicit" formula for the ratio of the kernel determinants.

Proposition 4.1. We have

$$
\frac{\operatorname{det} \widehat{\mathscr{L}}_{T}}{\operatorname{det} \mathscr{L}_{T}}=\prod_{k=1}^{\operatorname{dim} \operatorname{ker}(\widehat{\mathscr{D}}+\widehat{T})} \int_{X}\left|\widehat{\phi}_{k}\right|^{2} d g .
$$

Proof. Recall from Theorem 2.2 that $\left.\operatorname{ker}\left(\mathscr{D}_{T}\right) \equiv \operatorname{ker}(\widehat{\mathscr{D}}+\widehat{T})\right|_{X}$; in particular, $\left\{a_{k} \widehat{\phi}_{k}\right\}$ is an orthonormal basis for ker $\mathscr{D}_{T}$, where $\left\{\widehat{\phi}_{k}\right\}$ an orthonormal basis for $\operatorname{ker}(\widehat{\mathscr{D}}+\widehat{T})$ and $a_{k}:=1 /\left\|\widehat{\phi}_{k}\right\|_{X}$ with $\left\|\widehat{\phi}_{k}\right\|_{X}^{2}:=\int_{X}\left|\widehat{\phi}_{k}\right|^{2} d g$. Therefore, setting $v_{k}:=\gamma_{0} \widehat{\phi}_{k}$, we have

$$
\mathscr{L}_{T}:=\sum_{k=1}^{h_{T}} a_{k}^{2} v_{k} \otimes v_{k}, \quad \widehat{\mathscr{L}}_{T}:=\sum_{k=1}^{h_{T}} v_{k} \otimes v_{k},
$$

where $h_{T}=\operatorname{dim} \operatorname{ker}(\widehat{D}+\widehat{T})=\operatorname{dim} \operatorname{ker} \mathscr{D}_{T}$. Now with respect to the basis $\left\{v_{k}\right\}$, we can write

$$
\mathscr{L}_{T}=\left(\begin{array}{cccc}
a_{1}^{2}\left\langle v_{1}, v_{1}\right\rangle & a_{1}^{2}\left\langle v_{2}, v_{1}\right\rangle & \cdots & a_{1}^{2}\left\langle v_{h_{T}}, v_{1}\right\rangle \\
a_{2}^{2}\left\langle v_{1}, v_{2}\right\rangle & a_{2}^{2}\left\langle v_{2}, v_{2}\right\rangle & \cdots & a_{2}^{2}\left\langle v_{h_{T}}, v_{2}\right\rangle \\
\vdots & \vdots & \ddots & \vdots \\
a_{h_{T}}^{2}\left\langle v_{1}, v_{h_{T}}\right\rangle & a_{h_{T}}^{2}\left\langle v_{2}, v_{h_{T}}\right\rangle & \cdots & a_{h_{T}}^{2}\left\langle v_{h_{T}}, v_{h_{T}}\right\rangle
\end{array}\right)
$$

where $\langle$,$\rangle denotes the L^{2}$ inner product on $Y$. It follows that

$$
\operatorname{det} \mathscr{L}_{T}=a_{1}^{2} \cdots a_{h_{T}}^{2} \cdot \operatorname{det} \widehat{\mathscr{L}}_{T}=\left(\prod_{k=1}^{h_{T}}\left\|\widehat{\phi}_{k}\right\|_{X}^{2}\right)^{-1} \cdot \operatorname{det} \widehat{\mathscr{L}}_{T},
$$

and this formula implies our result. 


\section{Acknowledgment}

The authors thank the referee for corrections and helpful suggestions, all of which considerably improved this paper.

\section{References}

[Atiyah et al. 1975] M. F. Atiyah, V. K. Patodi, and I. M. Singer, "Spectral asymmetry and Riemannian geometry, I", Math. Proc. Cambridge Philos. Soc. 77 (1975), 43-69. MR 53 \#1655a Zbl 0297.58008

[Atiyah et al. 1976] M. F. Atiyah, V. K. Patodi, and I. M. Singer, "Spectral asymmetry and Riemannian geometry, III", Math. Proc. Cambridge Philos. Soc. 79:1 (1976), 71-99. MR 53 \#1655c Zbl 0325.58015

[Brüning and Lesch 1999] J. Brüning and M. Lesch, "On the $\eta$-invariant of certain nonlocal boundary value problems”, Duke Math. J. 96:2 (1999), 425-468. MR 99m:58180 Zbl 0956.58014

[Bunke 1995] U. Bunke, "On the gluing problem for the $\eta$-invariant", J. Differential Geom. 41:2 (1995), 397-448. MR 96c:58163 Zbl 0821.58037

[Dai and Freed 1994] X. Dai and D. S. Freed, " $\eta$-invariants and determinant lines", J. Math. Phys. 35:10 (1994), 5155-5194. MR 96a:58204 Zbl 0822.58048

[Douglas and Wojciechowski 1991] R. G. Douglas and K. P. Wojciechowski, "Adiabatic limits of the $\eta$-invariants. The odd-dimensional Atiyah-Patodi-Singer problem”, Comm. Math. Phys. 142:1 (1991), 139-168. MR 92j:58110 Zbl 0746.58074

[Grubb and Seeley 1996] G. Grubb and R. T. Seeley, "Zeta and eta functions for Atiyah-PatodiSinger operators”, J. Geom. Anal. 6:1 (1996), 31-77. MR 97g:58174 Zbl 0858.58050

[Hassell et al. 1995] A. Hassell, R. Mazzeo, and R. B. Melrose, "Analytic surgery and the accumulation of eigenvalues", Comm. Anal. Geom. 3:1-2 (1995), 115-222. MR 97f:58132 Zbl 0854.58039

[Kirk and Lesch 2004] P. Kirk and M. Lesch, "The $\eta$-invariant, Maslov index, and spectral flow for Dirac-type operators on manifolds with boundary", Forum Mathematicum 16:4 (2004), 553-629. MR 2005b:58029 Zbl 02157957

[Lesch and Wojciechowski 1996] M. Lesch and K. P. Wojciechowski, "On the $\eta$-invariant of generalized Atiyah-Patodi-Singer boundary value problems”, Illinois J. Math. 40:1 (1996), 30-46. MR 97d:58194 Zbl 0866.58054

[Loya 2005] P. Loya, "Dirac operators, boundary value problems, and the $b$-calculus", pp. 241280 in Spectral geometry of manifolds with boundary and decomposition of manifolds (Roskilde, Denmark, 2003), edited by B. Booß-Bavnbek et al., Contemp. Math. 366, Amer. Math. Soc., Providence, RI, 2005. MR 2005k:58038 Zbl 1065.58017

[Loya and Melrose 2003] P. Loya and R. Melrose, "Fredholm perturbations of Dirac operators on manifolds with corners", Preprint, 2003.

[Loya and Park 2004] P. Loya and J. Park, "The $\zeta$-determinant of generalized APS boundary problems over the cylinder", J. Phys. A 37:29 (2004), 7381-7392. MR 2005f:58060 Zbl 1062.58035

[Loya and Park 2005] P. Loya and J. Park, "On the gluing problem for Dirac operators on manifolds with cylindrical ends", J. Geom. Anal. 15:2 (2005), 285-319. MR MR2152484

[Loya and Park 2006] P. Loya and J. Park, "On the gluing problem for the spectral invariants of Dirac operators", Adv. Math. (2006). To appear.

[Mazzeo and Melrose 1995] R. R. Mazzeo and R. B. Melrose, "Analytic surgery and the eta invariant”, Geom. Funct. Anal. 5:1 (1995), 14-75. MR 96a:58200 Zbl 0838.57022 
[Mazzeo and Piazza 1998] R. Mazzeo and P. Piazza, "Dirac operators, heat kernels and microlocal analysis. II. Analytic surgery", Rend. Mat. Appl. (7) 18:2 (1998), 221-288. MR 2000a:58065 Zbl 0926.58017

[Melrose 1993] R. B. Melrose, The Atiyah-Patodi-Singer index theorem, Research Notes in Mathematics 4, A K Peters, Wellesley, MA, 1993. MR 96g:58180 Zbl 0796.58050

[Melrose and Piazza 1997] R. B. Melrose and P. Piazza, "Families of Dirac operators, boundaries and the $b$-calculus", J. Differential Geom. 46:1 (1997), 99-180. MR 99a:58144 Zbl 0955.58020

[Müller 1996] W. Müller, "On the $L^{2}$-index of Dirac operators on manifolds with corners of codimension two. I”, J. Differential Geom. 44:1 (1996), 97-177. MR 98b:58163 Zbl 0881.58071

[Nicolaescu 1995] L. I. Nicolaescu, "The Maslov index, the spectral flow, and decompositions of manifolds”, Duke Math. J. 80:2 (1995), 485-533. MR 96k:58208 Zbl 0849.58064

[Scott 2002] S. Scott, "Zeta determinants on manifolds with boundary", J. Funct. Anal. 192:1 (2002), 112-185. MR 2003g:58051 Zbl 1007.58018

[Scott and Wojciechowski 2000] S. G. Scott and K. P. Wojciechowski, "The $\zeta$-determinant and Quillen determinant for a Dirac operator on a manifold with boundary", Geom. Funct. Anal. 10:5 (2000), 1202-1236. MR 2001k:58067 Zbl 0973.58017

[Wojciechowski 1995] K. P. Wojciechowski, "The additivity of the $\eta$-invariant: the case of a singular tangential operator", Comm. Math. Phys. 169:2 (1995), 315-327. MR 96k:58210 Zbl 0837.58030

Received July 5, 2004. Revised October 10, 2004.

\author{
PAUL LOYA \\ DEPARTMENT OF MATHEMATICS \\ BINGHAMTON UNIVERSITY \\ VESTAL PARKWAY EAST \\ BINGHAMTON, NY 13902 \\ UNITED STATES \\ paul@math.binghamton.edu \\ JINSUNG PARK \\ SCHOOL OF MATHEMATICS \\ Korea InSTITUTE FOR AdVANCED STUdy \\ 207-43, CHEONGNYANGNI 2-DONG \\ DONGDAEMUN-GU \\ SEOUL 130-722 \\ KOREA \\ jinsung@kias.re.kr
}

